\title{
Achieve Research Continuity During Social Distancing by Rapidly Implementing Individual and Group Videoconferencing with Participants: Key Considerations, Best Practices, and Protocols
}

\author{
Stephanie Marhefka ${ }^{1}$ Elizabeth Lockhart ${ }^{1} \cdot$ DeAnne Turner $^{2}$
}

Published online: 2 April 2020

(c) Springer Science+Business Media, LLC, part of Springer Nature 2020

\begin{abstract}
The novel coronavirus has upended many traditional research procedures as universities and other research entities have closed to activate social distancing. Some social and behavioral research activities (e.g. data analysis, manuscript preparation) can be continued from other environments with appropriate security protocols in place. For studies involving in-person interactions, continuity may be more difficult. Phone-based interactions provide a low-tech solution that may suffice in some cases. Yet, videoconferencing platforms can nearly replicate in-person interactions, activating both auditory and visual senses and potentially resulting in more substantial engagement. Staff can meet with participants individually or in groups, each seeing and hearing one another in real time. This paper provides guidance for researchers transitioning in-person assessments and interventions to a synchronous videoconferencing platform. Best practices, key considerations, examples from the field, and sample protocols are presented to ease transition for ongoing studies and maximize the potential of videoconferencing —and social distancing.
\end{abstract}

Keywords Social distancing · Videoconferencing · Intervention research · ehealth

\section{Introduction}

Currently, much of the world is practicing social distancing to mitigate the risk of the novel coronavirus spreading beyond our healthcare capacity [1]. While not as concerning as the lives that have been and will be lost due to this pandemic, social and behavioral HIV researchers are facing a situation that could stall or hinder data collection and intervention delivery. The health impacts of COVID-19 on participants and staff will likely affect many projects, but mhealth research projects will likely be less disrupted, as their platforms and interactions are largely impervious to

Electronic supplementary material The online version of this article (https://doi.org/10.1007/s10461-020-02837-x) contains supplementary material, which is available to authorized users.

Stephanie Marhefka

smarkhefk@usf.edu

1 College of Public Health, University of South Florida, 13201 Bruce B. Downs Blvd., MDC 56, Tampa, FL 33612, USA

2 Center for Interdisciplinary Research on AIDS, Yale University School of Public Health, New Haven, CT, USA social distancing measures. Ongoing research projects that have relied on in-person contact may be in jeopardy as people self-isolate, and as in-person data collection has been banned or discouraged by some institutions. This is problematic for achieving research objectives-especially considering the pandemic and related social distancing practices may continue for months. Stopping studies or data collection may also have unexpected negative impacts on participants. Participants living with and those at-risk for HIV who are currently enrolled in research may rely, in part, on remuneration from research participation to help with costs of food and housing. Additionally, the social connections participants experience from taking part in research may be especially valuable to their mental health-particularly during this pandemic, which is anxiety-inducing and isolating.

Videoconferencing offers a valuable solution in this critical time. Over the past 11 years, our team has been studying videoconferencing as a means of delivering interventions to people living with HIV (PLWH) [2-8]. During that time, we have adapted programs to the videoconferencing platform and engaged in one-on-one as well as group-based videoconferencing interactions for research $[4,6,8]$. We have obtained promising findings regarding the feasibility, 
acceptability and potential efficacy of videoconferencingadapted versions of the group-based Healthy Relationships intervention and a tobacco cessation program - both specific to PLWH $[4,6]$. We have also engaged in focus groups and individual assessments over videoconferencing, resulting in quantitative and qualitative data to understand experiences and nuances $[4,6]$. Moreover, our work has helped us to understand access to internet and devices needed for videoconferencing (i.e., "smart-phones," tablets, desktops or laptop computers) and potential willingness to engage in videoconferencing individually and in groups $[2,3,5$, 9]. These experiences provide the basis for this manuscript, which reports on processes and considerations for rapidly implementing videoconferencing to connect with participants for data collection, as well as for individual and groupbased interventions. While spurred by the need for project continuity during this public health crisis, this article may also be beneficial for researchers who are planning to commence a study employing videoconferencing in the future.

\section{Methodological Considerations}

\section{How can Videoconferencing Best Fit your Project?}

Research teams should assess the potential fit between videoconferencing, their project goals, participants, and staff.

\section{For Which Projects will Videoconferencing Work?}

For non-intervention studies that rely on self-report paper or computer-based questionnaires that participants read and complete independently, videoconferencing may be unnecessary; online survey platforms should meet the needs of such studies. However, if the protocol involves staff reading aloud to participants and recording the selected responses, or if an open-ended interview is involved, videoconferencing could be a great option. Screen sharing options enable participants to read along, which may speed the process. The ability to view participants' faces and body language could help the staff when a participant is silent due to confusion, contemplation, or distraction. Additionally, the ability of participants to see the staff member may increase engagement and attention.

For individual or group-based intervention studies, videoconferencing serves to mimic the face-to-face interactions in many ways [8]. Participants can see and hear one another in real time, which may help with group cohesion. For interventions specific to living with HIV-or even those which involve discussing sexual risk behaviors with persons not living with HIV, seeing other's reactions to personal stories and revelations can make it much easier to share. When we initially adapted Healthy Relationships for videoconferencing, prior Healthy Relationships participants said they would miss "the hugs" [5]. Yet, in our work we found participants developed ways to successfully demonstrate their affection for group members, including putting their hands into a heart shape to communicate warmth. Additionally, the videoconferencing setting typically allows for screen sharing, which means content can be shared with participants in real time. We have implemented a virtual whiteboard [8] simply by opening a document and typing key points during individual or group interaction. While individual or group-based interventions could be delivered via phone if not in person, the visual connection provides interpersonal and educational advantages.

\section{For Which Participants will Videoconferencing Work?}

If you are considering incorporating videoconferencing, it will be important to acknowledge that-as with many strategies-this will not work for everyone. Although "smart phones" are common, in a recent study [9] we found that $30.3 \%$ of a large sample of PLWH in Florida reported that they did not have access to a phone with internet [9]. 13.6\% did not have internet at home via any device [9]. In qualitative interviews, some PLWH reported sharing phones with friends or roommates, which could pose challenges. Others reported having limited data plans that might preclude videoconferencing. Most (94\%) of those who had the required technology were willing to engage in videoconferencing related to HIV, but not everyone was willing [9]. If you decide to use videoconferencing, to reduce inequities and maximize the reach of your work, consider alternative ways to engage those who cannot or will not use this medium [9]. Flexibility is advised.

\section{Which Staff are a Good Fit for Videoconferencing Engagement?}

Not every staff member will be the best fit for videoconferencing assessments or intervention delivery. Some people may be uncomfortable managing the technology or seeing their face on the screen. When we were adapting Healthy Relationships for videoconferencing [8], we had a community advisory board member, who had extensively facilitated Healthy Relationships in-person, facilitate a mock session over videoconferencing. Despite being a comfortable, professional, and engaging facilitator at his community organization, he froze in front of the videoconferencing camera. We recommend having facilitators and staff practice videoconferencing engagement before graduating them to videoconferencing sessions with participants; those who are uncomfortable with videoconferencing may need to be shifted to other tasks. 


\section{Where can Participants and Staff be When they Engage in Video-Calls?}

Many people have the capability of joining videoconferencing from nearly anywhere [10], but the need for privacy and confidentiality means clear protocols for research participation are critical. Individual video-calls may necessitate fewer requirements than group video-calls. For individual engagement with staff, we recommend participants attend while in a "private space" without distractions (people, pets, etc.) and sit in a room in which they can speak freely about HIV or other topics. If the space is noisy, we suggest they use headphones or ear buds. For our video-group interactions, we have always required a "private space", but have learned concrete rules are necessary (See Supplemental File
1). In our work, when we saw another person walking in the background of one participant's screen during a video-group session, we learned that it was important to define "private space." Having other people in the background means persons not part of the group could see and hear other group members [6], constituting privacy breeches that diminish trust in the process and the research team. If participants believe their privacy is being compromised, they may leave the group or not actively participate. We now require participants to join from a locked room in which they are the only person. Staff should also engage from private spaces with headphones or earbuds. If others must be in the room (i.e., you have an observer with the facilitator) the person should be introduced to the participant(s). See further text and Tables 1, 2, 3 and Supplemental Files 1 and 3-5 for best

Table 1 Key considerations for choosing a platform

\begin{tabular}{ll}
\hline Accessible & What devices (e.g., phone, tablet, computer) and operating systems support the platform (or do not)? \\
& How much bandwidth is required to run the platform? \\
User friendly & Is it easy for participants to join? Can participants access the platform without downloading a program? \\
& Can participants access the platform free of charge? \\
& What types of technical support are available (e.g., written support, chats, "how-to" video(s)? \\
Available & What features are needed to conduct your research? \\
Features & Does the platform have: chat functions, polling, extra rooms, facilitator/host controls, recording, screen/document sharing, \\
& range of viewing options (how do those differ based on viewing device), muting options? \\
What are the limits to the number of simultaneous participants or co-occurring video sessions? & Does your institution have licenses for a viable videoconferencing platform that would work for your project at no addi- \\
tional cost? & What charges can you expect per platform? Do any features require additional fees? \\
Appropriate Security & Is end-to-end encryption available? Is there an associated fee? \\
& What methods can the host use to ensure only participants have access to the session (e.g., meeting passwords, ability to \\
limit who enters the session or preventing additional participants from entering)? & What protections are available to prevent participants from recording the meeting or using screen capture? \\
& If needed, are HIPAA-compliant settings available? Is an additional fee or agreement required?
\end{tabular}

Table 2 Rationale for selected features of videoconferencing platforms

\begin{tabular}{ll}
\hline Bandwidth Required & - Can impact a participant's ability to view videos or participate in real time discussion \\
& - Important consideration if participants have low internet speed or shared internet \\
User accounts & - May act as an extra security measure depending on the participants/study \\
& - For participants, remembering usernames and passwords can pose an extra, possibly unnecessary, layer of difficulty \\
- Using a URL (delivered via email) to directly access meetings has been vital for people of all technology abilities to & participate in our research \\
Screen sharing & - May be important if the research requires participants to see and interact with different materials \\
& - Researchers may need to screen share with participants if their intervention typically refers to slides, handouts, videos, \\
& and/or pictures \\
Host controls & - Can limit/grant access and override participant controls (most useful for managing participation in group-based (vs. \\
& one-on-one) videoconferencing) \\
& - May enable facilitators/staff to mute participants whose background noise interferes with group sessions \\
& - May allow facilitators/staff to remove permissions for participants to privately chat with each other, which help \\
& maintain focus \\
- Used to assess fidelity of the intervention session curricula & Removes need for real-time intervention observers for supervision and fidelity assessment \\
- Caution: storing this recording may increase privacy risks
\end{tabular}


Table 3 Facilitator/staff best practices
1. Environment

2. Professionalism

3. Managing challenges
- In general, follow the same privacy rules/guidelines as participants

- Privacy is critical—regardless of whether at work or elsewhere

- Be alone in a room with a locked door

- Do not join from public spaces

- Use only password-protected internet connections

- If another person must be present for training or quality assurance purposes, introduce that person to participant(s) at call onset and explain why they are present

- Remove any private, controversial, or other information from the wall behind you (it could be visible on the screen) to limit distracting and/or offending participants

- If there are others in the space outside of your door, consider putting a sign on the door or talking with them to request a quiet environment

- Maintain a professional appearance; wear professional attire

- Be prepared for challenges that may arise

- Have technical support manuals or guides on hand. Even if staff are comfortable with the software, participants may encounter challenges with their devices or with using the platform

- Especially for group calls, have a staff member on call to enable the facilitator to continue while a participant is receiving technical assistance

- Have a contingency plan. What will happen if the connection is unsuccessful for one or more participants? Will you allow some or all join via phone instead? practices, guidelines, and protocols for maintaining privacy in the video-group setting.

\section{How can my Team Implement Videoconferencing?}

Here, we have some suggestions about how to proceed once you decide videoconferencing is the best solution for your project.

Ensure Technical Capacity Depending on the platform, your staff and participants will likely have the option of participating from a variety of devices. You will need to ensure an adequate internet connection (may vary based on platform) and devices with working cameras, and microphones (internal or external to the device). As noted, headphones and earbuds should be secured to maximize privacy-your staff may already have their own.

Choose a Platform When transitioning to a videoconferencing software, it is important to assess fit between the platform and the project needs (Table 1). Choose a platform that is user friendly and accessible by both research staff and participants. If participants do not find the platform easy to use, participation may decline. Depending on the nature of the study, required interactions between research staff and participants, and required interactions between participants in group-based interventions, the features needed to suc- cessfully transition an in-person study to videoconferencing may vary. Although not comprehensive, Table 2. delineates reasons to carefully consider which features are available in the platform you select.

The security of the videoconferencing platform, especially when working with PWLH, may be the most important feature to consider. Steps being taken to ensure security should be clearly communicated with participants in simple language (See Supplemental File 2). Check with your institution about whether additional security assurances may be needed (e.g., HIPAA compliance, written agreements). The availability of these measures differs by platform and may require additional levels of administrative approval.

Our team has experience using a variety of videoconferencing platforms for research and non-research purposes and have settled on Zoom $®$, for its accessibility across devices and operating systems, ease of use for participants with no or limited videoconferencing experience, performance in low-bandwidth situations, screen-sharing features, host controls, and security settings. Our participants report satisfaction with the platform and even those with no prior videoconferencing experience have been able to use Zoom with ease [6]. As the videoconferencing market has experienced substantial growth in recent years, many alternatives are available and may be conducive to research, depending on characteristics of the population under study. Each team should identify the best fit for their project(s). 
Confirm Granting Agency Approval and Institutional Com-

pliance Before transitioning to videoconferencing, check with funders and institutional review boards. In this unprecedented time of social distancing, we expect support for using alternative approaches to achieve research continuity, but assure that approvals are in place before you pivot. Emailing a program/project officer may be sufficient for receiving funder approvals. Determine if rapid review by the institutional review board (IRB) is possible. Information about platform security and other videoconferencing-specific measures to promote participant privacy (See Table 3 and Supplemental File 1) should be communicated to IRBs and included in the research consent process. Note that for future studies it may be useful to include contingency plans with initial IRB submissions for in-person studies to allow for transitioning to videoconferencing or other online options if the need arises.

Develop Protocols for Videoconferencing Implementation Once an appropriate and user-friendly platform is identified, engaging with participants via that platform should be relatively straightforward. Having protocols in place will simplify the process and help reduce unexpected challenges.

Protocols for Facilitators and staff should include standards for promoting privacy and confidentiality, professionalism, and responses to challenges. For considerations, see Table 3. For a sample protocol you can adapt for your project, see Supplemental File 3.

Protocols for Participants may include instructions for setting up the videoconferencing platform and, depending on platform, downloading software before first use. We have found when participants test out the platform individually in advance of any formal sessions, it alleviates participant anxiety and reduces complications during formal sessions. See Supplemental File 4. for a sample videoconferencing testing protocol.

Whether conducting individual or group-based videosessions, ground rules are helpful in promoting appropriate interactions. Ground rules can foster focused participation, comfort, and group cohesion; they can also help to avert uncomfortable situations (Supplemental Files $1 \& 5$ ).

\section{Revise Study Materials and Procedures for the Video-Call}

Environment Depending on the purpose for using videoconferencing in research, it may be helpful to revise study materials. If your team is planning to use videoconferencing to complete a structured or semi-structured interview that had formally been conducted in person, revisions may be limited to introducing participants to the videoconferencing environment and ensuring participation from a space in which their privacy is unlikely to be compromised. However, if you were previously conducting in-person group ses- sions and now plan to deliver them over video-groups, some processes may need to be changed.

When we adapted the Healthy Relationships program for video group delivery [8], it required changing the way certain components were delivered while still maintaining the core elements. For example, Healthy Relationships in-person involves some "fish bowl" activities, in which two participants sit in the center of a circle and role play conversations related to HIV status disclosure and sexual risk reduction. We were able to maintain the activity but the implementation was altered. While video-group participants cannot sit around a room together, they can see and hear one another in real time. In this study, we took advantage of the way our platform always showed the person who was speaking in a large box on the screen, and that the person who was speaking never saw their own face in that large box (instead, they saw the last person who spoke before themselves). Facilitators asked the person who would respond to the main speaker (we'll call her Diana) to say one word to cue the large box and asked the main speaker (let's call him Robert) to then begin the role play. This enabled Diana to see Robert's face in the large box during the dialogue, while Robert saw Diana's face at that same time (the others saw the person currently speaking in the large box). In doing this, Robert could feel more like he was speaking directly with Diana, while, Diana would feel as if she was speaking directly with Robert.

Participant materials were distributed differently in our Healthy Relationships adaptation vs. in-person groups. Upon consent to do so, staff mailed or emailed handouts to participants and showed handouts via screen share as appropriate during the sessions. In a tobacco cessation program for PLWH, handouts were mailed and/or emailed depending on the participant's preference. Researchers working with PLWH should assess the feasibility of distributing handouts via mail or email, which could compromise privacy if the handouts reveal participants' HIV status - especially for participants residing with, or sharing email addresses with, people unaware the participant is living with HIV.

Biological specimen collection is a challenge for research with only online interactions. Creative solutions were needed when we began a pilot study of a video-group tobacco cessation intervention for PLWH and required biochemical verification of tobacco abstinence. To address this, we developed a strategy to send oral swab tests for cotinine (measuring presence of nicotine) to participants and then guide them through the process during individual video-calls [6]. The video component enabled our team to ensure proper swab technique, ensure the swab reflected the participant's nicotine levels (vs. those of their dog or roommate), and help the participant prepare the packet for the express shipping service. This strategy could be adopted for analyses involving other specimens. For example, a video-call could be used 
to support participants in collecting dried blood spots for analysis [11, 12].

Create Meaningful Interactions It is possible to create meaningful interactions when hosting meetings or sessions via videoconferencing. Existing guidance for videoconferencing etiquette $[13,14]$ can be applied in both individual and group-based interventions. A simple suggestion for staff/facilitators is to maintain eye contact with the camera. Although a small action, this effort can contribute to meaningful connections with research participants. In our work, participants have noted the connection they felt with staff and fellow participants. Relatedly, across intervention groups and studies, participants have developed hand gestures to express compassion. In a study of video-groups among women living with HIV, women made a fist with one hand, then held that wrist with their opposite hand and raised their arms just over their heads to indicate a hug. Similarly, to show support in a video-group tobacco cessation study, participants made the shape of a heart with their hands to show support. Despite not interacting with their peers in-person, participants developed strong social support and cohesion (e.g., "That was what really brought us back, is, you know, the, the friendships that we have built here in, in talking to each other. And, and you actually, like, look forward to, like, oh, you know, I can't wait till the next session... "-participant of a tobacco cessation program for people living with HIV).

\section{Help Participants Prepare for the Video-Call Experience Data} from one of our recent studies suggests many PLWH may not have engaged in videoconferencing previously [9]. In our projects, lack of prior experience has not been problematic when participants were provided with instructions and assistance from our team [6]. For teams new to using videoconferencing for research, it may be helpful to provide participants and staff video tutorials like this one provided by Zoom before the first video-call with a participant [15]. Other platforms have video tutorials available, as well [16, 17]. Alternatively, you may wish to create your own.

Our team assesses a person's ability to join a videoconference as part of eligibility screening. We start with a phone call, and guide participants through the connection process, ultimately stopping the phone conversation and finishing the interaction on the videoconference. On the occasion that a potential participant has trouble connecting, we do our best to help them problem-solve. Persons who are unable to make the connection are disqualified. However, as noted above, teams transitioning to videoconferencing due to social distancing practices may instead allow such participants to participate via other technologies. For video-groups, based on feedback received in early focus groups [5], we would caution against allowing some participants to join via audio only while other participants in the same session are showing their video. As noted, those showing their live video feed may feel especially vulnerable and unwilling to speak frankly if they cannot see some group members' live feed. Instead, participants unable to join a videoconference may be given the option of an individual encounter via phone or joining a group that involves phone/audio interactions exclusively.

Consider Pandemic-Specific Needs, as Appropriate During this pandemic, research staff should be cognizant of the many stressors participants may be facing. Like much of the world, it is likely that at least some research participants will become ill with COVID-19. Teams should be familiar with local guidance for seeking medical attention for possible COVID-19 to inform participants if needed. Although the primary role of the research team is not to provide pandemic-specific social support, teams should have lists of local referral sources, as appropriate, to assist them in connecting participants with basic needs (e.g., food, housing) as well as other supportive services (e.g., mental health).

\section{Summary}

During times of social distancing and beyond, researchers conducting social-behavioral studies may want to transition their research to videoconferencing, which many participants and staff can join from their own homes. Consideration will need to be given to what projects are most appropriate and feasible to be transitioned to videoconferencing, which is the best fit for their study, how to prepare participants and staff, how to ensure support from institutions and funders, and how to create meaningful interactions. If done thoughtfully, the transition to videoconferencing can help ensure the continuity of research and provide a way for participants to benefit from potentially meaningful social interaction during a time of isolation.

\section{References}

1. Wilder-Smith A, Freedman D. Isolation, quarantine, social distancing and community containment: pivotal role for old-style public health measures in the novel coronavirus $(2019-\mathrm{nCoV})$ outbreak. J Travel Med. 2020. https://doi.org/10.1093/jtm/taaa0 20.

2. Green SM, Lockhart E, Marhefka SL. Advantages and disadvantages for receiving Internet-based HIV/AIDS interventions at home or at community-based organizations. AIDS Care. 2015;27(10):1304-8

3. Marhefka S, Turner D, Lockhart E. Understanding women's willingness to use ehealth for HIV-related services: a novel application of the technology readiness and acceptance model 
to highly stigmatized medical condition. Telemed J E Health. 2019;25(6):511-8.

4. Marhefka SL, Buhi ER, Baldwin J, Chen H, Johnson A, Lynn $\mathrm{V}$, et al. Effectiveness of healthy relationships video-group-a videoconferencing group intervention for women living with HIV: preliminary findings from a randomized controlled trial. Telemed J E Health. 2014;20(2):128-34.

5. Marhefka SL, Fuhrmann HJ, Gilliam P, Lopez B, Baldwin J. Interest in, concerns about, and preferences for potential video-group delivery of an effective behavioral intervention among women living with HIV. AIDS Behav. 2012;16(7):1961-9.

6. Marhefka SL, Turner D, Lockhart E, Rivara A, Wang W, Shuter J. Meeting our patients "where they are": video-group smoking cessation for people living with HIV. J Assoc Nurses AIDS Care. 2018;29(2):338-44.

7. Turner D, Lockhart E, Marhefka SL. Willingness of MSM living with HIV to take part in video-groups: application of the technology readiness and acceptance model. AIDS Behav. 2019;23(11):3165-74.

8. Marhefka SL, Iziduh S, Fuhrmann HJ, Lopez B, Glueckauf R, Lynn V, et al. Internet-based video-group delivery of healthy relationships-A "prevention with positives" intervention: report on a single group pilot test among women living with HIV. AIDS Care. 2013;25(7):904-9.

9. Marhefka SL, Lockhart E, Turner D, Wang W. Social determinants of potential eHealth engagement among people living with HIV receiving ryan white case management: health equity implications from project TECH. AIDS Behav. 2019. https://doi. org/10.1007/s10461-019-02723-1.

10. Ryan CL. Computer and internet use in the United States: 2016. US Department of Commerce, Economics and Statistics Administration, US, 2018 Contract No.: Report Number ACS 39.
11. Hirshfield S, Teran RA, Downing MJ Jr, Chiasson MA, Tieu H-V, Dize L, et al. Quantification of HIV-1 RNA among men who have sex with men using an at-home self-collected dried blood spot specimen: feasibility study. JMIR Public Health Surveill. 2018;4(4):e10847.

12. Ngo-Giang-Huong N, Khamduang W, Leurent B, Collins I, Nantasen I, Leechanachai P, et al. Early HIV-1 diagnosis using in-house real time PCR amplification on dried blood spots for infants in remote and resource limited settings. J Acquir Immune Defic Syndr. 2008;49(5):465.

13. Inc. ZVC. Video Communications Best Practice Guide 2020. https://blog.zoom.us/wordpress/2018/07/03/video-communicat ions-best-practice-guide/. Accessed 16 March 2020.

14. Northwestern University. Videoconferencing Tips for Success 2016. https://www.it.northwestern.edu/videoconferencing/tips. html. Accessed 16 March 2020

15. Zoom Video Communications Inc. Zoom Video Tutorials 2019. https://support.zoom.us/hc/en-us/articles/206618765-Zoom-Video -Tutorials. Accessed 15 March 2020

16. Microsoft. Getting started 2020. https://support.skype.com/en/ skype/all/start/. Accessed 16 March 2020

17. LogMeIn Inc. GoToMeeting Quick Guide for Attendees 2020. https://www.gotomeeting.com/meeting/resources/gotomeetin g-quick-and-helpful-guide-for-attendees. Accessed 16 March 2020

Publisher's Note Springer Nature remains neutral with regard to jurisdictional claims in published maps and institutional affiliations. 